\title{
Spectral Methods and a Maximum Principle
}

\author{
By Claudio Canuto*
}

\begin{abstract}
Various spectral Chebyshev approximations of a model boundary layer problem for both a Helmholtz and an advection-diffusion operator are considered. It is assumed that simultaneously the boundary layer width tends to zero and the resolution power of the numerical method tends to infinity. The behavior of the spectral solutions in the frequency space and in the physical space is investigated. Error estimates are derived.
\end{abstract}

0. Introduction. Spectral methods using expansions in eigenfunctions of singular Sturm-Liouville operators (such as Chebyshev or Legendre polynomials) have been proven successful in the numerical approximation of various boundary value problems (see, e.g., [3], [8], [14] and the references therein).

Among the features of these methods is the possibility of accurately representing boundary layers. Such a property is related to the high resolution power of the spectral basis functions near the boundary points, since it is there where they concentrate most of their extrema (see [8, Section 3]). Using a suitable coordinate transformation it is even possible to achieve infinite-order accuracy near the boundaries [13].

In this paper we analyze the behavior of various spectral approximations to the following model boundary value problems:

$$
\left\{\begin{array}{lc}
-\varepsilon U_{x x}+\mathscr{L} U=0, & -1<x<1, \\
U(-1)=0, & U(1)=1,
\end{array}\right.
$$

where $\varepsilon>0$ is a constant and either $\mathscr{L} U \equiv U$ (as a model for a Helmholtz problem) or $\mathscr{L} U=U_{x}$ (as a model for an advection-diffusion problem). The spectral solution $u$ is a global polynomial of degree $N$, expanded in terms of Chebyshev polynomials and defined by any of the discrete procedures popularly used in spectral methods, namely a Galerkin or a tau or a collocation scheme. We shall establish several properties of $u$ which hold uniformly with respect to $\varepsilon$ and $N$, and we shall derive estimates on the error $u-U$ as $\varepsilon \rightarrow 0$ and $N \rightarrow \infty$ simultaneously.

The exact solution $U$ is uniformly bounded between 0 and 1 by the classical maximum principle (for complete studies on singular perturbation problems we refer, e.g., to [7], [10]). In contrast, the spectral solution is not positive throughout the domain if $\varepsilon$ is so small compared to $N^{-1}$ that a Gibbs phenomenon occurs at $x=1$. In other words, the maximum principle in the physical space does not

Received December 16, 1985; revised August 24, 1987.

1980 Mathematics Subject Classification (1985 Revision). Primary 65N30; Secondary 65N35.

* Part of this work was done while the author was visiting the School of Mathematics and the Institute for Mathematics and its Applications at the University of Minnesota in the Winter of 1985. 
hold for spectral approximations, as indeed is expected for high-order methods. However, an interesting conclusion of the present investigation is that a sort of "maximum principle" may hold in the frequency space, in the sense that all the Chebyshev coefficients of the spectral solution $u$ are strictly positive for all $\varepsilon$ and $N$.

The essence of this property is the fact that the Chebyshev coefficients of any derivative of $u$ depend linearly upon the Chebyshev coefficients of $u$ via a matrix with nonnegative entries. Thus, the positivity of $u$ in the frequency space can be proved by showing that $u$ is the exact solution of the equation $-\varepsilon u_{x x}+\mathscr{L} u=\Phi$, where $\Phi$ is a suitable polynomial of degree $N$ whose Chebyshev coefficients are positive.

Remarkably, the "maximum principle" in the frequency space easily implies the uniform boundedness of $u$ in the physical space. Hence, the property pointed out in this paper could be invoked to get a priori estimates in the maximum norm in more complex problems. Note that all the smoothing operators commonly used in order to filter out in the frequency space the spurious oscillations generated by sharp gradients and discontinuities (see, e.g., [9], [11]) are sign-preserving in the frequency space (i.e., the smoothing factors are nonnegative). Thus, the results presented here hold for filtered solutions as well. Their extension to more general situations deserves further investigation.

Our analysis also suggests that as $\varepsilon$ becomes very small compared to $N^{-1}$, spectral approximations of boundary layer problems may exhibit a strong sensitivity to the parity of $N$. For the model problems considered here, $u$ behaves better (in terms of the magnitude of oscillations) if it corresponds to an odd $N$ rather than to an even $N$.

Our results can easily be extended to other spectral bases such as the Legendre polynomials. Applications have been made to the numerical analysis of the KleiserSchumann algorithm for the Stokes problem [5] and the Schwarz alternating method for solving elliptic problems in complex geometries [2].

1. Notation and Basic Properties of the Chebyshev Orthogonal System. Throughout the paper, given two integers $m$ and $k$, we will use the following notation:

$$
k \rtimes m \quad \text { if and only if } \quad|k-m| \text { is even. }
$$

Conversely, $k \ngtr m$ if and only if $|k-m|$ is odd.

For each integer $N>0, \mathbf{P}_{N}$ will denote the space of real polynomials on the real line of degree at most $N . \stackrel{\circ}{\mathbf{P}}_{N}$ will denote the subspace of $\mathbf{P}_{N}$ of polynomials which vanish at $x= \pm 1$.

Each polynomial $v \in \mathbf{P}_{N}$ can be expanded in terms of Chebyshev polynomials of the first kind, $T_{m}(x)=\cos m \theta$ (if $\cos \theta=x$ ), as follows:

$$
v=\sum_{m=0}^{N} \hat{v}_{m} T_{m}
$$

Since the Chebyshev polynomials satisfy the orthogonality relation

$$
\int_{-1}^{1} T_{m}(x) T_{l}(x) \omega(x)=\frac{\pi}{2} c_{m} \delta_{m l},
$$


where $\omega(x)=1 / \sqrt{1-x^{2}}$ is the Chebyshev weight and

$$
c_{m}= \begin{cases}2, & m=0 \\ 1, & m>0\end{cases}
$$

we have

$$
\hat{v}_{m}=\left(\frac{2}{\pi c_{m}}\right)^{1 / 2} \int_{-1}^{1} v(x) T_{m}(x) \omega(x) d x .
$$

The Chebyshev expansion of the first derivative $v_{x}$ of $v$ is given by

$$
v_{x}=\sum_{m=0}^{N-1} \hat{v}_{m}^{(1)} T_{m}
$$

where

$$
\hat{v}_{m}^{(1)}=\frac{2}{c_{m}} \sum_{\substack{k \geq m+1 \\ k \supset \mathfrak{k} m}} k \hat{v}_{k} .
$$

Similarly, the expansion of the second derivative is given by

$$
v_{x x}=\sum_{m=0}^{N-2} \hat{v}_{m}^{(2)} T_{m}
$$

where

$$
\hat{v}_{m}^{(2)}=\frac{1}{c_{m}} \sum_{\substack{k \geq m+2 \\ k \supset \subset m}} k\left(k^{2}-m^{2}\right) \hat{v}_{k}
$$

We recall that the Chebyshev polynomials satisfy the differential equation

$$
\left(\sqrt{1-x^{2}} T_{m}^{\prime}(x)\right)^{\prime}+\frac{m^{2}}{\sqrt{1-x^{2}}} T_{m}(x)=0, \quad m=0,1, \ldots ;
$$

they form a complete orthogonal basis in the Hilbert space

$$
L_{\omega}^{2}(-1,1)=\left\{v:(-1,1) \rightarrow \mathbf{R} \text { measurable such that } \int_{-1}^{1} v^{2}(x) \omega(x) d x<+\infty\right\}
$$

with the inner product $(u, v)_{\omega}=\int_{-1}^{1} u(x) v(x) \omega(x) d x$. We will denote by $\|v\|_{0, \omega}=$ $\sqrt{(v, v)_{\omega}}$ the norm in $L_{\omega}^{2}(-1,1)$, and more generally by

$$
\|v\|_{r, \omega}=\left(\sum_{k=0}^{r}\left\|\frac{d^{k} v}{d x^{k}}\right\|_{0, \omega}^{2}\right)^{1 / 2}
$$

the norm of the weighted Sobolev space $H_{\omega}^{r}(-1,1)$ of the functions whose distributional derivatives up to order $r$ are in $L_{\omega}^{2}(-1,1)$.

2. Spectral Methods for a Helmholtz Equation. In this section we shall investigate different approximations of spectral type for the following boundary value problem:

$$
\begin{cases}-\varepsilon U_{x x}+U=0, & -1<x<1, \varepsilon>0, \\ U(-1)=0, & U(+1)=1 .\end{cases}
$$

The exact solution is $U(x)=\sinh ((x+1) / \sqrt{\varepsilon}) / \sinh (2 / \sqrt{\varepsilon})$, which exhibits a boundary layer of width $O(\sqrt{\varepsilon})$ near $x=1$ as $\varepsilon \rightarrow 0$. 
Problem (2.1) can be discretized spectrally using a Galerkin, or a tau, or a collocation procedure (see, e.g., [8], [3]). For each integer $N>0$, the spectral solution is a polynomial of degree $N$ satisfying the boundary conditions in (2.1), i.e,

$$
u \in \mathbf{P}_{N}, \quad u(-1)=0, \quad u(+1)=1
$$

and one of the following sets of equations:

(A) Galerkin method

$$
\left(-\varepsilon u_{x x}+u, v\right)_{\omega}=0 \quad \forall v \in \stackrel{\circ}{\mathbf{P}}_{N}
$$

(B) Tau method

$$
\left(-\varepsilon u_{x x}+u, v\right)_{\omega}=0 \quad \forall v \in \mathbf{P}_{N-2}
$$

(C) Collocation method

$$
\left(-\varepsilon u_{x x}+u\right)\left(x_{j}\right)=0, \quad j=1, \ldots, N-1,
$$

where

$$
x_{j}=\cos \frac{j \pi}{N}
$$

are the knots of the Gauss-Lobatto quadrature rule for the Chebyshev weight $\omega$.

For all fixed $\varepsilon>0$, each of the above approximations is stable and convergent as $N \rightarrow \infty$ (see [3, Chapter 10]). The rate of convergence is exponential in $N$ since the solution of $(2.1)$ is analytic.

2.1. Sign Properties of the Chebyshev Coefficients of $u$. We are interested in deriving estimates on the spectral solutions which hold uniformly as $\varepsilon \rightarrow 0$. To this end, let us first investigate for each of the above approximations the structure of the error equation, i.e., the differential equation satisfied exactly by the spectral solution.

THEOREM 2.1. Let $u$ be defined by (2.2) and one of the relations (2.3)-(2.5). Then $u$ satisfies the error equation

$$
-\varepsilon u_{x x}+u=\lambda \Phi_{N}+\mu \Phi_{N-1}, \quad-1<x<1,
$$

for suitable constants $\lambda, \mu \in \mathbf{R}$ and polynomials $\Phi_{n}$ (depending upon the method) such that

$$
\left\{\begin{array}{l}
\operatorname{deg} \Phi_{n}=n, \\
\text { parity of } \Phi_{n}=\text { parity of } n, \\
\left(\hat{\Phi}_{n}\right)_{k} \geq 0, \quad 0 \leq k \leq n .
\end{array}\right.
$$

Proof. Let us set $L u=-\varepsilon u_{x x}+u . \quad L u$ is a polynomial of degree $N$ which can be expanded according to a basis in $\mathbf{P}_{N}$. For the Galerkin method (2.3) it is convenient to use the basis defined by

$$
\Phi_{n}=T_{n+1}^{\prime}, \quad n=0, \ldots, N .
$$

On the other hand, a basis for $\dot{\mathbf{P}}_{N}$ is given by

$$
\Psi_{l}=\left(1-x^{2}\right) T_{l+1}^{\prime}, \quad l=0, \ldots, N-2 .
$$


The two bases satisfy the orthogonality relation

$$
\begin{aligned}
\left(\Phi_{n}, \Psi_{l}\right)_{\omega} & =\int_{-1}^{1} \sqrt{1-x^{2}} T_{n+1}^{\prime} T_{l+1}^{\prime} d x=-\int_{-1}^{1}\left(\sqrt{1-x^{2}} T_{n+1}^{\prime}\right)^{\prime} T_{l+1} d x \\
& =-(n+1)^{2} \int_{-1}^{1} T_{n+1} T_{l+1} \frac{d x}{\sqrt{1-x^{2}}}=-\frac{\pi}{2}(n+1)^{2} \delta_{n, l} .
\end{aligned}
$$

Here we have used Eq. (1.9). Then (2.7) follows from (2.3), and (2.8) is an immediate consequence of the definition (2.9), taking into account (1.6). For the tau method (2.4), the natural basis for $\mathbf{P}_{N}$ is the Chebyshev basis

$$
\Phi_{n}=T_{n},
$$

so that both (2.7) and (2.8) are straightforward. Finally, consider the collocation method (2.5). Since both $L u$ and $T_{N}^{\prime}$ vanish at the interior nodes $x_{j}, j=1, \ldots$, $N-1$, one has

$$
L u=(\lambda x+\mu) T_{N}^{\prime}
$$

Hence, (2.7) holds if we set

$$
\Phi_{N}=x T_{N}^{\prime}, \quad \Phi_{N-1}=T_{N}^{\prime} .
$$

In order to conclude, we have to check that the Chebyshev coefficients of $\Phi_{N}$ are nonnegative. By (1.6) one has

$$
x T_{N}^{\prime}=\sum_{k=0}^{N-1} b_{k} x T_{k}
$$

where $b_{k} \geq 0$. The third property in (2.8) therefore follows from the identities $x T_{0}=T_{1}$ and $x T_{k}=\cos \theta \cos k \theta=\frac{1}{2}\left(T_{k+1}+T_{k-1}\right)$ for $k \geq 1(\theta=\arccos x)$.

The information on the sign of the Chebyshev coefficients of the error polynomials $\Phi_{n}(n=N, N-1)$ can be used to infer the positivity of the Chebyshev coefficients of the spectral solutions.

THEOREM 2.2. Let $u=\sum_{m=0}^{N} \hat{u}_{m} T_{m}$ be the Chebyshev expansion of $u$. Then

$$
\hat{u}_{m}>0, \quad m=0, \ldots, N \text {. }
$$

Proof. First note that for a given polynomial $\Phi \in \mathbf{P}_{N}$, there exists a unique polynomial $v \in \mathbf{P}_{N}$ such that

$$
-\varepsilon v_{x x}+v=\Phi
$$

The Chebyshev coefficients of $v$ are recursively defined by the relations

$$
-\varepsilon \hat{v}_{m}^{(2)}+\hat{v}_{m}=\hat{\Phi}_{m}, \quad m=N, N-1, \ldots, 0
$$

i.e., recalling (1.8),

$$
\hat{v}_{m}=\hat{\Phi}_{m}+\varepsilon \frac{1}{c_{m}} \sum_{\substack{k \geq m+2 \\ k>m}} k\left(k^{2}-m^{2}\right) \hat{v}_{k}, \quad m=N, N-1, \ldots, 0 .
$$

This relation shows that the $\hat{v}_{m}$ 's are nonnegative if the $\hat{\boldsymbol{\Phi}}_{m}$ 's are. Let us now define $v \in \mathbf{P}_{N}$ by the identity

$$
-\varepsilon v_{x x}+v=\Phi_{N}
$$


One has by (2.8) and (2.16),

$$
\begin{cases}\hat{v}_{m}>0 & \text { if } m \propto N \\ \hat{v}_{m}=0 & \text { otherwise }\end{cases}
$$

Similarly, if we define $w \in \mathbf{P}_{N}$ by

$$
-\varepsilon w_{x x}+w=\Phi_{N-1}
$$

we have

$$
\begin{cases}\hat{w}_{m}=0 & \text { if } m \propto N \\ \hat{w}_{m}>0 & \text { otherwise. }\end{cases}
$$

By (2.7), the spectral solution $u$ can be represented as

$$
u=\lambda v+\mu w,
$$

where $\lambda$ and $\mu$ are obtained by imposing the boundary conditions. Specifically, one has

$$
\left\{\begin{array}{l}
\lambda\left(\sum_{k \supset N} \hat{v}_{k}\right)+\mu\left(\sum_{k \supset \subset N} \hat{w}_{k}\right)=1, \\
\lambda\left(\sum_{k \supset N} \hat{v}_{k}\right)-\mu\left(\sum_{k \supset \subset N} \hat{w}_{k}\right)=0,
\end{array}\right.
$$

whence

$$
\left\{\begin{array}{l}
\lambda=\left[2 \sum_{k \supset N} \hat{v}_{k}\right]^{-1}>0 \\
\mu=\left[2 \sum_{k \supset K^{\prime} N} \hat{w}_{k}\right]^{-1}>0
\end{array}\right.
$$

We conclude that

$$
\hat{u}_{m}= \begin{cases}\lambda \hat{v}_{m} & \text { if } m \propto N, \\ \mu \hat{w}_{m} & \text { if } m \propto N,\end{cases}
$$

proving the theorem.

Remark 2.1. The above proof contains a constructive procedure for computing the spectral solution of the boundary value problem (2.1). If the coefficients $\hat{v}_{m}$ and $\hat{w}_{m}$ are computed by obvious recurrence relations, the method requires $O(N)$ operations.

The result stated in Theorem 2.2 can be regarded as a "maximum principle" in the frequency space for the spectral approximations of the boundary value problem (2.1). Unlike the exact solution, which is strictly positive in the open interval $(-1,1)$ for all $\varepsilon>0$, the spectral solution is not bounded from below by 0 if $\varepsilon$ is small enough compared to $N^{-1}$. A Gibbs phenomenon at $x=1$ produces oscillations around the $x$-axis. However, the lack of positivity of $u$ in the physical space is compensated for by the strict positivity of $u$ in the frequency space, in the sense of (2.13). In turn, this property can be used to derive information independent of $\varepsilon$ on the behavior of $u$ in the physical space. The following theorem is an example. 
THEOREM 2.3. For all $\varepsilon>0$ and all $N>0$ the following bound holds:

$$
|u(x)| \leq 1, \quad-1<x<1 .
$$

Moreover,

$$
|u(x)|=1 \quad \text { if and only if } \quad x=1
$$

Proof. One has

$$
\begin{aligned}
|u(x)| & =\left|\sum_{m=0}^{N} \hat{u}_{m} T_{m}(x)\right| \\
& \leq \sum_{m=0}^{N} \hat{u}_{m}\left|T_{m}(x)\right| \quad \text { by }(2.13) \\
& \leq \sum_{m=0}^{N} \hat{u}_{m}=\sum_{m=0}^{N} \hat{u}_{m} T_{m}(1)=u(1)=1 .
\end{aligned}
$$

If $|u(x)|=1$ then $x \neq-1$ and $\left|T_{m}(x)\right|=1$ for all $m$, hence $x=1$.

2.2. Asymptotic Behavior of $u$ as $\varepsilon \rightarrow 0, N \rightarrow \infty$. In this subsection we study the behavior of the error between the spectral solution $u$ and the exact solution $U$ of $(2.1)$, when both $N \rightarrow \infty$ and $\varepsilon \rightarrow 0$. The question is: How small can $\varepsilon$ be (compared to $N^{-1}$ ) in order that the error vanish in a suitable $L^{p}$-norm?

First, let us briefly review the information given by the energy method, in the simplest case of the Galerkin scheme (2.3).

THEOREM 2.4. The following estimates hold:

$$
\begin{gathered}
\varepsilon\left\|u_{x}-U_{x}\right\|_{0, \omega}^{2} \leq C\left\{\frac{N^{-2}}{\varepsilon}+\frac{N^{-4}}{\varepsilon^{2}}\right\}, \\
\|u-U\|_{0, \omega}^{2} \leq C^{\prime}\left\{\frac{N^{-2}}{\varepsilon}+\frac{N^{-4}}{\varepsilon^{2}}\right\}^{2} .
\end{gathered}
$$

Proof. Recall ([4]) that the bilinear form

$$
a(u, v)=-\int_{-1}^{1} u_{x x} v \omega d x
$$

is continuous and coercive over $H_{\omega, 0}^{1}(-1,1)=H_{\omega}^{1}(-1,1) \cap H_{0}^{1}(-1,1)$, i.e., there exist strictly positive constants $\alpha$ and $\beta$ such that

$$
\begin{cases}a(u, u) \geq \alpha\|u\|_{1, \omega}^{2} & \forall u \in H_{\omega, 0}^{1}(-1,1), \\ |a(u, v)| \leq \beta\|u\|_{1, \omega}\|v\|_{1, \omega} & \forall u, v \in H_{\omega, 0}^{1}(-1,1) .\end{cases}
$$

Then the well-known error estimate for the Galerkin method (see, e.g., [6]) reads as follows:

$$
\begin{aligned}
& \varepsilon\left\|u_{x}-U_{x}\right\|_{0, \omega}^{2}+\|u-U\|_{0, \omega}^{2} \\
& \quad \leq \frac{\beta}{\alpha} \inf _{\substack{\phi \in \mathbf{P}_{N} \\
U-\phi \in H_{\omega, 0}^{1}(-1,1)}}\left\{\varepsilon\left\|U_{x}-\phi_{x}\right\|_{0, \omega}^{2}+\|U-\phi\|_{0, \omega}^{2}\right\} \\
& \quad \leq \frac{\beta}{\alpha} \inf _{\Psi \in \dot{\mathbf{P}}_{N}}\left\{\varepsilon\left\|\tilde{U}_{x}-\Psi_{x}\right\|_{0, \omega}^{2}+\|\tilde{U}-\Psi\|_{0, \omega}^{2}\right\}
\end{aligned}
$$


where $\tilde{U}=U-(x+1) / 2$. It has been proven in [12] that there exists $\bar{\Psi} \in \stackrel{\circ}{\mathbf{P}}_{N}$ such that

$$
\left\|\tilde{U}_{x}-\bar{\Psi}_{x}\right\|_{0, \omega}+N\|\tilde{U}-\bar{\Psi}\|_{0, \omega} \leq C N^{-1}\|\tilde{U}\|_{2, \omega} .
$$

Since $\|\tilde{U}\|_{2, \omega} \simeq O\left(\varepsilon^{-1}\right)$, we obtain from (2.28)

$$
\varepsilon\left\|u_{x}-U_{x}\right\|_{0, \omega}^{2}+\|u-U\|_{0, \omega}^{2} \leq C\left\{\frac{N^{-2}}{\varepsilon}+\frac{N^{-4}}{\varepsilon^{2}}\right\} .
$$

The estimates in the $L_{\omega}^{2}$-norm can be improved using the Aubin-Nitsche argument (see, e.g., [6, Theorem 3.2.4]).

The theorem implies that the error $u-U$ vanishes in the $L_{\omega}^{2}$-norm if $\varepsilon N^{2} \rightarrow \infty$ as $N \rightarrow \infty$. However, this is not the best condition guaranteeing convergence. A sharper analysis can be carried out using the results derived in the previous subsections. The theorems we will prove are as follows:

THEOREM 2.5. Let $u$ be the spectral approximation of problem (2.1) produced either by the Galerkin method (2.3) or by the collocation method (2.5). There exist constants $c_{1}$ and $c_{2}$ independent of $\varepsilon$ and $N$ such that

$$
\begin{gathered}
\|u-U\|_{0, \omega} \leq c_{1} \min \left(\frac{1}{N^{1 / 2}}, \frac{1}{\varepsilon N^{4+1 / 2}}\right), \\
\|u-U\|_{L^{\infty}(-1,1)} \leq c_{2} \min \left(1, \frac{1}{\varepsilon N^{4}}\right) .
\end{gathered}
$$

It follows that $u-U$ vanishes in the $L_{\omega}^{2}$-norm, as $N \rightarrow \infty$, no matter how small $\varepsilon$ is, and in the maximum norm provided $\varepsilon N^{4} \rightarrow \infty$ as $N \rightarrow \infty$.

THEOREM 2.6. Let $u$ be the spectral approximation of problem (2.1) produced by the tau method (2.4). There exists a constant $C$ independent of $\varepsilon$ and $N$ such that

$$
\|u-U\|_{L^{\infty}(-1,1)} \leq \frac{C}{\varepsilon N^{4}}
$$

The $L_{\omega}^{2}$-norm of $u-U$ has the same asymptotic behavior.

Proof of Theorem 2.5. The error $z=u-U$ satisfies by (2.1) and (2.7) the following boundary value problem:

$$
\left\{\begin{array}{l}
-\varepsilon z_{x x}+z=\lambda \Phi_{N}+\mu \Phi_{N-1}, \quad-1<x<1 \\
z(1)=z(-1)=0
\end{array}\right.
$$

If we multiply by $z$ and integrate over $(-1,1)$ with respect to the Chebyshev weight $\omega$ we obtain

$$
\sqrt{\varepsilon}\left\|z_{x}\right\|_{0, \omega}+\|z\|_{0, \omega} \leq C\left\{|\lambda|\left\|\Phi_{N}\right\|_{0, \omega}+|\mu|\left\|\Phi_{N-1}\right\|_{0, \omega}\right\} .
$$

Multiplying, instead, by $|z|^{p-2} z(1<p<+\infty)$ and integrating over $(-1,1)$, we get the estimate

$$
(p-1) \varepsilon \int_{-1}^{1}|z|^{p-2} z_{x}^{2} d x+\left(\int_{-1}^{1}|z|^{p} d x\right)^{1 / p} \leq\left(\int_{-1}^{1}\left|\lambda \Phi_{N}+\mu \Phi_{N-1}\right|^{p} d x\right)^{1 / p} .
$$

Letting $p \rightarrow \infty$, we obtain

$$
\|z\|_{L^{\infty}(-1,1)} \leq|\lambda|\left\|\Phi_{N}\right\|_{L^{\infty}(-1,1)}+|\mu|\left\|\Phi_{N-1}\right\|_{L^{\infty}(-1,1)} .
$$


Since the $L_{\omega}^{2}$ - or $L^{\infty}$-norms of $\Phi_{N}$ and $\Phi_{N-1}$ can be easily estimated in terms of $N$, we only have to investigate the behavior of $\lambda$ and $\mu$, which are given by the formula (2.23), as functions of $\varepsilon$ and $N$.

In the Galerkin method, $\Phi_{N}$ and $\Phi_{N-1}$ are given by (2.9). Using (1.6) we have $\left\|T_{n}^{\prime}\right\|_{0, \omega} \simeq O\left(n^{3 / 2}\right.$ ), while (see, e.g., [8, Formula A.5]) $\max _{-1 \leq x \leq 1}\left|T_{n}^{\prime}(x)\right|=$ $T_{n}^{\prime}(1)=n^{2}$. In order to derive an estimate for $\lambda$, let us consider (2.16) for $m \propto N$. We have $\hat{v}_{m} \geq\left(\hat{\Phi}_{N}\right)_{m}=2 N / c_{m}$, which in turn gives

$$
\hat{v}_{m} \geq \frac{2 N}{c_{m}}+\frac{4 \varepsilon N}{c_{m}^{2}} \sum_{\substack{k=m+2 \\ k \ngtr N}}^{N} k\left(k^{2}-m^{2}\right) .
$$

Summing up over the indices $m \propto N$, it is easily seen that there exist constants $c_{1}$ and $c_{2}$ such that

$$
\lambda^{-1}=2 \sum_{m \supset N} \hat{v}_{m} \geq c_{1} N^{2}+c_{2} \varepsilon N^{6}
$$

Hence,

$$
|\lambda| \|\left.\Phi_{N}\right|_{0, \omega} \leq C /\left(c_{1} N^{1 / 2}+c_{2} \varepsilon N^{4+1 / 2}\right),
$$

while

$$
|\lambda|\left\|\Phi_{N}\right\|_{L^{\infty}} \leq C^{\prime} /\left(c_{1}+c_{2} \varepsilon N^{4}\right) .
$$

A similar estimate can be derived for $\mu \Phi_{N-1}$.

In the collocation method, $\Phi_{N}$ and $\Phi_{N-1}$ are given by (2.12). The $L_{\omega^{-}}^{2}$ and $L^{\infty}$ norms of $\Phi_{N}$ behave asymptotically as the corresponding norms of $T_{N+1}^{\prime}$, and the same is true for the Chebyshev coefficients of $\Phi_{N}$ with respect to the coefficients of $T_{N+1}^{\prime}$. It follows that the estimates for the Galerkin method apply to this case as well.

Proof of Theorem 2.6. Again we use (2.30) and (2.31). In this case, $\Phi_{N}=T_{N}$, $\Phi_{N-1}=T_{N-1}$, hence both their $L_{\omega^{-}}^{2}$ and $L^{\infty}$-norms are $O(1)$. By (2.16) we have $\hat{v}_{m} \geq\left(2 \varepsilon / c_{m}\right) N\left(N^{2}-m^{2}\right)$ for all $m \rtimes N, m<N$. Hence,

$$
\lambda^{-1}=2 \sum_{m \ngtr N} \hat{v}_{m} \geq 4 \varepsilon N \sum_{\substack{m \ngtr N \\ m<N}} \frac{N^{2}-m^{2}}{c_{m}} \geq C \varepsilon N^{4}
$$

for a suitable constant $C>0$. A similar estimate holds for $\mu^{-1}$.

Remark 2.2. The previous results can be considered optimal. Indeed, one can easily prove that, for the Galerkin method, $\lambda, \mu \geq C N^{-2}$ if $\varepsilon N^{4} \leq C_{0}$, where $C_{0}$ is a sufficiently small constant. In this case, $\|u-U\|_{L^{\infty}(-1,1)}$ is uniformly bounded away from zero. A similar behavior occurs for the tau and the collocation methods.

3. Spectral Methods for an Advection-Diffusion Equation. We discuss now spectral methods for the following boundary value problem:

$$
\begin{cases}-\varepsilon U_{x x}+U_{x}=0, & -1<x<1, \varepsilon>0, \\ U(-1)=0, & U(1)=1 .\end{cases}
$$

The exact solution is $U(x)=\left(e^{(x+1) / 2}-1\right) /\left(e^{2 / \varepsilon}-1\right)$. The boundary layer exhibited near $x=1$ when $\varepsilon \rightarrow 0$ now has a width of order $O(\varepsilon)$. The spectral approximation is defined again by $(2.2)$ and a projection procedure. We consider here the two 
following schemes:

(A) Galerkin (collocation) method

$$
\left(-\varepsilon u_{x x}+u_{x}, v\right)_{\omega}=0 \quad \forall v \in \stackrel{\circ}{\mathbf{P}}_{N}
$$

(B) Tau method

$$
\left(-\varepsilon u_{x x}+u_{x}, v\right)=0 \quad \forall v \in \mathbf{P}_{N-2} .
$$

Note that the Galerkin and the collocation method coincide for problem (3.1) since $-\varepsilon u_{x x}+u_{x} \in \mathbf{P}_{N-1}$ if $u \in \mathbf{P}_{N}$ and the collocation nodes (2.6) are the knots of a quadrature rule which is exact for polynomials of degree up to $2 N-1$.

3.1. Sign Properties of the Chebyshev Coefficients of $u$. By adapting the proof of Theorem 2.1 one has the following result.

THEOREM 3.1. Let $u$ be defined by (2.2) and one of the relations (3.2) or (3.3). Then $u$ satisfies the error equation

$$
-\varepsilon u_{x x}+u_{x}=\mu \Phi_{N-1}
$$

where $\mu \in \mathbf{R}$ is a suitable constant and $\Phi_{N-1}$ satisfies (2.8) (more precisely, $\Phi_{N-1}=T_{N}^{\prime}$ for the Galerkin method, $\Phi_{N-1}=T_{N-1}$ for the tau method).

In order to analyze the Chebyshev expansion of $u$, let us define $v$ as the unique polynomial of degree $N-1$ such that

$$
-\varepsilon v_{x}+v=\Phi_{N-1} .
$$

LEMMA 3.1. The Chebyshev coefficients of the polynomial $v$ defined by (3.5) satisfy

$$
\hat{v}_{m}>0 \quad \text { for } m=0, \ldots, N-1 \text {. }
$$

The proof of the lemma is similar to the proof of Theorem 2.2 ; hence, it will be omitted.

Next let $w=\sum_{m=1}^{N} \hat{w}_{m} T_{m}$ be the unique polynomial of degree $N$ such that $w_{x}=v$ and $\int_{-1}^{1} w(x)\left(1-x^{2}\right)^{-1 / 2} d x=0$. The spectral solution $u$ can be expressed as

$$
u(x)=\lambda+\mu w(x), \quad-1 \leq x \leq 1 .
$$

By imposing the boundary conditions (2.2) one gets

$$
\begin{gathered}
\mu=\frac{1}{2 \sum_{k \text { odd }} \hat{w}_{k}}, \\
\lambda=\frac{1}{2}\left[\begin{array}{c}
\sum_{\substack{k \text { even } \\
k \neq 0}} \hat{w}_{k} \\
\sum_{k \text { odd }} \hat{w}_{k}
\end{array}\right] .
\end{gathered}
$$

In order to discuss the behavior of the Chebyshev coefficients of $u$, we have to distinguish whether $u$ is defined by a Galerkin (collocation) or a tau method. Let us consider the former case first.

(A) Galerkin (collocation) method. We first present the most significant results of this section, then we sketch their proofs. 
THEOREM 3.2. Let $u=\sum_{m=0}^{N} \hat{u}_{m} T_{m}$ be the solution of (2.2) and (3.2). Then

$$
\hat{u}_{m}>0 \text { for } m=1, \ldots, N
$$

and

$$
u(x) \leq 1 \quad \text { for }-1 \leq x \leq 1
$$

When $\varepsilon$ is small compared to $N^{-1}$, a sharper result can be given. More precisely, we assume that

$$
\varepsilon \leq C_{0} N^{-2} \quad \text { as } N \rightarrow \infty
$$

for a suitable constant $C_{0}>0$. Note that when $C_{0}$ is small enough, the expansion does not resolve the boundary layer.

THEOREM 3.3. Let $u=\sum_{m=0}^{N} \hat{u}_{m} T_{m}$ be the solution of (2.2) and (3.2). Assume that (3.12) holds for a constant $C_{0}$ small enough.

(a) If $N$ is odd, then

$$
\hat{u}_{m}>0 \quad \text { for } m=0, \ldots, N
$$

hence $|u(x)| \leq 1$ for all $x,|x| \leq 1$. Moreover,

$$
u \simeq \frac{1}{2}+\frac{1}{2} T_{N} \quad \text { as } \varepsilon \rightarrow 0, \varepsilon \ll N^{-2} .
$$

(b) If $N$ is even, then

$$
\left\{\begin{array}{l}
\hat{u}_{0}<0 \\
\hat{u}_{m}>0, \quad m=1, \ldots, N .
\end{array}\right.
$$

Moreover, if $\varepsilon \ll N^{-2}$ then $\left|\hat{u}_{0}\right| \simeq \hat{u}_{N}=O\left(\varepsilon N^{2}\right)^{-1}$, hence $u$ is not bounded from below independently of $\varepsilon$. More precisely,

$$
u \simeq \hat{u}_{0}+\hat{u}_{N} T_{N} \quad \text { as } \varepsilon \rightarrow 0, \varepsilon \ll N^{-2} .
$$

Remark 3.1. The right-hand side of (3.14) is an even function. Since $u$ has to satisfy the boundary conditions (2.2), the odd coefficients of $u$, though negligible with respect to $\hat{u}_{0}$ and $\hat{u}_{N}$, produce a nonnegligible contribution near the endpoints.

Remark 3.2. For very small $\varepsilon$ the spectral solution corresponding to an odd $N$ behaves better than the one produced by an even $N$ of the same magnitude. From a practical point of view this means that for a given $\varepsilon$ the oscillations created by the boundary layer will be less pronounced if the discretization parameter $N$ is chosen to be odd. Although this phenomenon may be related to the particular structure of the boundary layer problem considered in (3.1), the interesting implication in the previous analysis is that attention should be paid to the parity of the degree of the polynomials to be used in a spectral approximation of boundary layer problems.

Let us now sketch the proofs of the previous results.

Proof of Theorem 3.2. By (3.5) and (1.6), the Chebyshev coefficients of $v$ satisfy the recurrence relation

$$
\hat{v}_{m}=\frac{2 \varepsilon}{c_{m}} \sum_{\substack{k \geq m+1 \\ k \supset \mathbb{k} m}} k \hat{v}_{k}+\hat{\Phi}_{m}, \quad m=N-1, N-2, \ldots, 0,
$$


where the Chebyshev coefficients $\hat{\Phi}_{m}$ of $\Phi_{N-1}=T_{N}^{\prime}$ are given by

$$
\hat{\Phi}_{m}= \begin{cases}2 N / c_{m} & \text { if } m \propto N, \\ 0 & \text { otherwise. }\end{cases}
$$

On the other hand, the Chebyshev coefficients of $w$ satisfy

$$
\begin{gathered}
2 N \hat{w}_{N}=\hat{v}_{N-1}, \\
2 m \hat{w}_{m}=c_{m-1} \hat{v}_{m-1}-\hat{v}_{m+1}, \quad m=N-1, \ldots, 1 .
\end{gathered}
$$

From (3.17) and (3.15) we obtain $\hat{w}_{N}=1$, while (3.18) and (3.15) give

$$
\hat{w}_{m}=\varepsilon \hat{v}_{m}, \quad m=1, \ldots, N-1 \text {. }
$$

Multiplying (3.15) by $\varepsilon$ we obtain

$$
\left\{\begin{array}{l}
\hat{w}_{N}=1, \\
\hat{w}_{m}=\frac{2 \varepsilon}{c_{m}} \sum_{\substack{k \geq m+1 \\
k>k<m}} k \hat{w}_{k}+\frac{2 N \varepsilon}{c_{m}} \sigma_{m N}, \quad m \leq N-1,
\end{array}\right.
$$

where

$$
\sigma_{m N}= \begin{cases}1 & \text { if } m \not N \\ 0 & \text { otherwise }\end{cases}
$$

Thus, $\hat{w}_{m}>0$ for $m=1, \ldots, N$. Then $\mu>0$ by (3.8), hence (3.10) follows from (3.7). The inequality (3.11) can be derived by the same argument as used in the proof of Theorem 2.3 .

Proof of Theorem 3.3. Let us first state two technical results.

LEMMA 3.2. Assume that (3.12) holds. If $C_{0}$ is small enough, there exist two constants $c_{1}$ and $c_{2}$ independent of $\varepsilon$ and $N$ such that

$$
\begin{aligned}
c_{1} & \leq \sum_{m \ngtr N} \hat{w}_{m} \leq c_{2}, \\
c_{1} \varepsilon N^{2} & \leq \sum_{m \supset \mathcal{K} N} \hat{w}_{m} \leq c_{2} \varepsilon N^{2} .
\end{aligned}
$$

LEMMA 3.3. Under the hypothesis of the previous lemma one has

$$
\hat{w}_{m}=O(\varepsilon N), \quad m \Varangle N,
$$

and

$$
c(\varepsilon N)^{2} \leq \hat{w}_{m} \leq C \varepsilon^{2} N^{3}, \quad m \rtimes N, m \leq N-2,
$$

for two constants $c$ and $C$ independent of $\varepsilon$ and $N$.

The proofs of the two lemmas are based upon the identities (3.20). Since they are lengthy and quite technical, they will be omitted. The interested reader can find them in [1]. Now assume that $N$ is odd. By Lemma 3.2,

$$
\sum_{m \text { even }} \hat{w}_{m}<\sum_{m \text { odd }} \hat{w}_{m} \quad \text { provided } C_{0} \text { is small enough, }
$$

hence, by (3.9), $\hat{u}_{0}=\lambda>0$. Moreover, when $\varepsilon \rightarrow 0$ and $\varepsilon \ll N^{-2}$, one deduces from (3.7)-(3.9) and Lemma 3.3 that $\hat{u}_{0}, \hat{u}_{N} \rightarrow \frac{1}{2}$ while $\hat{u}_{m}=o(1), 1 \leq m \leq N-1$. On the other hand, if $N$ is even, (3.26) holds with the inequality sign reversed, hence $\hat{u}_{0}=\lambda<0$. We conclude the proof using again Lemma 3.3. 
(B) Tau method.

THEOREM 3.4. Let $u=\sum_{m=0}^{N} \hat{u}_{m} T_{m}$ be the solution of (2.2) and (3.3). If $\varepsilon^{2}>1 /(4(N-2)(N-1))$, then (3.10) and (3.11) hold.

THEOREM 3.5. Let $u=\sum_{m=0}^{N} \hat{u}_{m} T_{m}$ be the solution of (2.2) and (3.3). Assume that (3.12) holds for a constant $C_{0}$ small enough. Then the Chebyshev coefficients of $u$ have the following asymptotic behavior:

(a) If $N$ is even, then

$$
\left\{\begin{array}{l}
0<\hat{u}_{m}=O(1), \quad m \neq 0, N, N-2 \\
0<\hat{u}_{N}=O(\varepsilon N)^{-1} \\
0>\hat{u}_{N-2}=O(\varepsilon N)^{-1} \\
0<\hat{u}_{0}=O\left(\varepsilon N^{2}\right)^{-1}
\end{array}\right.
$$

(b) If $N$ is odd, then

$$
\left\{\begin{array}{l}
0>\hat{u}_{m}=O\left(\varepsilon N^{2}\right), \quad m \neq 0, N-2, N \\
0>\hat{u}_{N}=O(N) \\
0<\hat{u}_{N-2}=O(N), \\
0<\hat{u}_{0}=O(1) .
\end{array}\right.
$$

Thus, for both even and odd $N, u$ is not bounded uniformly with respect to $\varepsilon$, and it exhibits the asymptotic behavior

$$
u \simeq \hat{u}_{N} T_{N}+\hat{u}_{N-2} T_{N-2} .
$$

Remark 3.3. By comparing (3.27) and (3.28) note that again the spectral solution exhibits a better behavior for odd $N$ than for even $N$, in terms of the magnitude of the oscillations produced by the boundary layer.

Proof of Theorem 3.4. For the tau method, $\Phi_{N-1}=T_{N-1}$. Thus by (3.15),

$$
\left\{\begin{array}{l}
\hat{v}_{N-1}=1 \\
\hat{v}_{m}=\frac{2 \varepsilon}{c_{m}} \sum_{\substack{k \geq m+1 \\
k>1<m}} k \hat{v}_{k}, \quad m<N-1 .
\end{array}\right.
$$

Using again (3.17) and (3.18) one gets the following identities:

$$
\left\{\begin{array}{l}
\hat{w}_{N}=1 / 2 N \\
\hat{w}_{N-2}=\frac{4 \varepsilon^{2}(N-2)(N-1)-1}{2(N-2)} \\
\hat{w}_{m}=\varepsilon \hat{v}_{m} \quad \forall m \neq N, N-2 .
\end{array}\right.
$$

Therefore, $\hat{w}_{m}>0$ for $0 \leq m \leq N$, whence the result follows.

Proof of Theorem 3.5. The theorem is based on the two following technical results, whose proof can be found again in [1].

LEMMA 3.4. Assume that (3.12) holds. If $C_{0}$ is small enough, there exist two constants $C_{1}$ and $C_{2}$ independent of $\varepsilon$ and $N$ such that

$$
C_{1} \leq \sum_{m \supset \mathbb{K} N} \hat{v}_{m} \leq C_{2}
$$


LEMMA 3.5. Assume that (3.12) holds. If $C_{0}$ is small enough, there exists a constant $C_{3}$ independent of $\varepsilon$ and $N$ such that

$$
\sum_{\substack{m \leq N-4 \\ m \times N}} \hat{v}_{m} \leq C_{3}
$$

Using Lemma 3.4 and (3.31) we obtain

$$
C_{1} \varepsilon \leq \sum_{m \supset N} \hat{w}_{m} \leq C_{2} \varepsilon
$$

while by Lemma 3.5 and (3.31) we obtain

$$
\sum_{m \rtimes N} \hat{w}_{m} \simeq \frac{-1}{N^{2}} \quad \text { independently of } \varepsilon .
$$

Using these relations, one can prove that $\mu$ (defined by (3.8)) is strictly positive (resp., negative) if $N$ is even (resp., odd). Thus, (3.27) and (3.28) easily follow from (3.31) and (3.34). The rest of the theorem is a direct consequence of these estimates.

3.2. Asymptotic Behavior of $u$ as $N \rightarrow \infty, \varepsilon \rightarrow 0$. As in Subsection 2.2, we look for conditions on $\varepsilon$ and $N$ which guarantee that the error between $u$ and the exact solution $U$ of (3.1) vanishes in some $L^{p}$-norm as $\varepsilon \rightarrow 0$ and $N \rightarrow \infty$.

THEOREM 3.6. Let $u$ be the solution of (2.2) and either the Galerkin (collocation) method (3.2) or the tau method (3.3). If $\varepsilon N^{2} \rightarrow \infty$ as $N \rightarrow \infty$ and $\varepsilon \rightarrow 0$ in such a way that $\varepsilon N^{2}=N^{\alpha}$ for a suitable $\alpha>0$, then

$$
\|u-U\|_{L^{\infty}(-1,1)}=O\left(N^{-s}\right) \quad \text { for every } s>0 .
$$

Proof. By (3.4), the error $z=u-U$ is the solution of the boundary value problem

$$
\left\{\begin{array}{l}
-\varepsilon z_{x x}+z_{x}=\mu \Phi_{N-1}, \\
z(-1)=z(1)=0
\end{array}\right.
$$

Let us split the data $\mu \Phi_{N-1} \equiv f$ into $f=\tilde{f}+\bar{f}$, where $\int_{-1}^{1} \tilde{f}(x) d x=0$ and $\bar{f} \in \mathbf{R}$. Correspondingly, we split $z$ into $z=\tilde{z}+\bar{z}$. Integrating the identity $-\varepsilon \tilde{z}_{x x}+\tilde{z}_{x}=\tilde{f}$ over $(-1,1)$ yields $\tilde{z}_{x}(-1)=\tilde{z}_{x}(1)$. Multiplying the same identity by $\tilde{z}$ and integrating over $(-1,1)$ we obtain $\left\|\tilde{z}_{x}\right\|_{L^{2}(-1,1)} \leq\|\tilde{f}\|_{L^{2}(-1,1)}$. On the other hand, by direct computation, $\bar{z}(x)=\bar{f}\left[x+\operatorname{coth} \frac{1}{\varepsilon}-e^{x / \varepsilon} \sinh \frac{1}{\varepsilon}\right]$. We conclude that

$$
\|z\|_{L^{\infty}(-1,1)} \leq C|\mu|\left\|\Phi_{N}\right\|_{L^{2}(-1,1)}
$$

for a constant $C$ independent of $\varepsilon$ and $N$.

We now give an estimate of the right-hand side of (3.38) for the two spectral methods considered in this section. For the Galerkin method,

$$
\left\|\Phi_{N-1}\right\|_{L^{2}(-1,1)} \leq\left\|\Phi_{N-1}\right\|_{0, \omega}=\left\|T_{N}^{\prime}\right\|_{0, \omega} \leq C N^{3 / 2} .
$$

Using (3.20) in a recursive way, one can prove (see [1] for the technical details) that for each integer $p>0$ there exists a constant $C_{p}>0$ such that

$$
|\mu| \leq C_{p}\left(\varepsilon N^{2}\right)^{-p}
$$


whence (3.36) follows.

For the tau method, $\left\|\Phi_{N-1}\right\|_{L^{2}(-1,1)} \leq 1$. Using now (3.31) (see again [1]), one can prove that $\mu$ satisfies an estimate of the form

$$
|\mu| \leq C_{p} N^{2}\left(\varepsilon N^{2}\right)^{-p}
$$

for all $p>0$, whence again (3.36) follows.

Acknowledgment. The author wishes to thank the referee for suggesting many improvements in the form of this paper.

Dipartimento di Matematica

Università di Parma

Parma, Italy

Istituto di Analisi Numerica del C.N.R.

Corso Carlo Alberto, 5

27100 Pavia, Italy

1. C. CAnuto, Spectral Methods and a Maximum Principle, IAN-CNR Report n. 476, Pavia, Italy, 1986.

2. C. CANUTO \& D. FUnARO, "The Schwarz algorithm for spectral methods," SIAM J. Numer. Anal., v. 25, 1988, pp. 24-40.

3. C. Canuto, M. Y. Hussaini, A. Quarteroni \& T. Zang, Spectral Methods in Fluid Dynamics, Springer Series in Computational Physics, Springer-Verlag, New York, 1987.

4. C. CANUto \& A. QUARTERONi, "Spectral and pseudo-spectral methods for parabolic problems with nonperiodic boundary conditions," Calcolo, v. 18, 1981, pp. 197-217.

5. C. Canuto \& G. SACChi Landriani, "Analysis of the Kleiser-Schumann method," $N u$ mer. Math., v. 50, 1986, pp. 217-243.

6. Ph. G. Ciarlet, The Finite Element Method for Elliptic Problems, North-Holland, Amsterdam, 1978.

7. W. Eckhaus, Singular Perturbations, North-Holland, Amsterdam, 1973.

8. D. Gottlieb \& S. A. Orszag, Numerical Analysis of Spectral Methods: Theory and Applications, SIAM, Philadelphia, Pa., 1977.

9. H. O KREISS \& J. Oliger, "Stability of the Fourier method," SIAM J. Numer. Anal., v. 16, 1979 , pp. $421-433$.

10. J. L. Lions, Perturbations Singulières dans les Problèmes aux Limites et en Contrôle Optimal, Lecture Notes in Math., Vol. 323, Springer-Verlag, Berlin, 1973.

11. A. MAjda, J. MCDonough \& S. Osher, "The Fourier method for nonsmooth initial data," Math. Comp., v. 32, 1978, pp. 10411081.

12 Y. MADAY \& A. QUARTERONI, "Legendre and Chebyshev spectral approximations of Burgers' equation," Numer. Math., v. 37, 1981, pp. 321332.

13. S. A. Orszag \& M. Y IsRAELI, "Numerical simulation of viscous incompressible flows," Ann. Rev. Fluid Mech., v. 6, 1974, pp. 281318.

14. R. G. Voigt, D. Gottlieb \& M. Y. Hussaini (Eds.), Spectral Methods for Partial Differential Equations, SIAM, Philadelphia, Pa., 1984. 